Rentré en Europe souffrant, i] se fixa dans le midi de la France, et adressa au Gouvernement sa démission à la fin de $\mathbf{1 8 8 3}$, sans avoir, malheureusement, fait exécuter les plans qu'il avait conçus.

Possédant des connaissances encyclopédiques, ses travaux sont très nombreux et excessivement variés; ils embrassent la géographie physique, la géologie, la météorologie, la philosophie de la nature, l'astronomie.

Je ne m'attacherai ici qu'à ces derniers:

C'est dans les Astronomische Nachrichten qu'il débuta en 1843 et 1844 (Nos. 492 et 496), par des articles sur la lumière zodiacale et sur l'aberration; ce dernier travail eut l'honneur d'une réponse de J. Herschel (No. 520 ).

En 1853, il proposait une méthode pour la détermination de la latitude, de la longitude, de l'heure et de l'azimuth au moyen des observations de passages d'étoiles fondamentales dans deux verticaux (Mém. Acad. Belgique, t. XXV).

Il publia, en 1859 , un mémoire sur la détermination du rayon vecteur d'une planète nouvelle (Bull. Acad. Belgique, $2^{\mathrm{e}}$ série, t. VIII); et, en 1862 , une méthode pour mesturer la parallaxe horizontale des astres (Bull. acad. Belgique, $2^{\text {e }}$ série, t. XIII) méthode sur laquelle il est revenu en I $^{8} 7$ I (ib., t. XXXII), pour proposer l'héliometre de son invention.

Les astronomes connaissent cet héliomètre, au moyen duquel on a pu effectuer au Chili 606 pointés pendant le passage de Vénus sur le soleil; au Texas les nuages ont empêché l'observation pendant une partie notable du phénomène.

En $\mathbf{3} 865$, Houzeau a donné une méthode qui permet d'étudier à la fois les mouvements propres d'étoiles appartenant à deux groupes distincts, en renversant, par réflexion, l'un des groupes sur l'image directe de l'autre (Bull. Acad. Belgique, $\mathbf{2}^{\mathrm{e}}$ série, t. XX). Mais cette méthode n'a pas été mise à exécution.

Une note très intéressante $(1873)$ est celle (Bull. acad. Belgique, $2^{e}$ série, $t$. XXXVI) dans laquelle il déduit, avec une exactitude que l'on ne pouvait pas espérer de ce procédé, la direction du mouvement du système solaire dans l'espace de la composante commune à toutes les trajectoires des comètes qui pénètrent dans ce système.

Citons enfin ses belles observations sur la lumière zodiacale, qui ont paru dans son »Résumé de quelques observations astronomiques et météorologiques faites dans la zône surtempérée et entre les tropiquese (Mém. in $8^{\circ}$ de l'Acad. de Belgique, t. XXV).

Ses derniers travaux et les plus importants, travaux qui seront toujours consultés avec beaucoup de fruit dans tous les observatoires, et qui le mettent certainement au premier rang des bibliographes scientifiques contemporains, se rattachent tous à la grande oeuvre qu'il avait entreprise et qu'il n'a pu achever entièrement: la Bibliographie générale de l'astronomie.

Déjà dans les Annales de l'observatoire de Bruxelles, il en avait fait paraître la partie la plus pratiquement utile sous le titre de: Constantes de l'astronomie. Plus tard il étendit le cadre de cet ouvrage et publia son , Vade Mecum de l'astronome (1882).

Le tome II et la I ${ }^{\text {ère }}$ partie du tome $I^{\text {ex }}$ de la Bibliographie générale de l'astronomie» ont paru avec la collaboration de M. Lancaster; la $\mathbf{2}^{\mathrm{e}}$ partie du tome $I^{\text {er }}$ était en cours d'impression lorsque Houzeau fut atteint de la maladie qui l'emporta.

Jusque dans ces derniers temps, il continua à y travailler avec ardeur et surtout à donner à son collaborateur les instructions nécessaires pour l'achèvement complet du monumen tum perenne qu'il laissera après lui. I 2 Juillet.

Vers la fir du mois d'Avril, seulement, il dut absolument renoncer à tout travail; il s'éteignit le

F. Folie.

\title{
The variable star near 26 Cygni.
}

A letter just received from Mr. Baxendell informs me that the star near 26 Cygni detected here March $23^{\text {rd }} 1887$ was observed by him on July $24^{\text {th }}$ and $26^{\text {th }}$, again as 7.I mag. It seems therefore to be a long period variable star. It was looked for here 1887 Aug. I 4 and Dec. I7, but was certainly on these dates below 14.0 mag. Various observations were made in the spring upto June $I^{\text {Bt }}$ but it was indistinguishable from the numerous small stars in the neighbourhood. Mr. Baxendell looked for it on June $18^{\text {th }}$ but with the same result. The rise must have been there- fore very rapid. The period would seem to be $\frac{484 \pm}{n}$ days. Although I have no record of any observations between Aug. 14 and Dec. 17 I887, I feel pretty sure that it was observed several times between these dates. The value of $n$ would therefore seem to be more easily $I$ than 2 . The variation is from 7.I to 14.0 . The place of the star from Dun Echt Circular No. 143 is ( 1887.0 ):

$$
\alpha=19^{\mathrm{h}} 5^{8^{\mathrm{m}} 15^{\mathrm{s}} .28} \delta=+49^{\circ} 43^{\prime} 40^{\prime \prime} 4 \text {. }
$$

